\title{
Addressing dyslipidemic risk beyond LDL-cholesterol
}

\author{
Alan R. Tall,, David G. Thomas, ${ }^{1}$ Ainara G. Gonzalez-Cabodevilla, ${ }^{2}$ and Ira J. Goldberg ${ }^{2}$ \\ 'Division of Molecular Medicine, Department of Medicine, Columbia University, New York, New York, USA. ²Division of Endocrinology, Diabetes and Metabolism, Department of Medicine, New York University \\ Grossman School of Medicine, New York, New York, USA.
}

\begin{abstract}
Despite the success of LDL-lowering drugs in reducing cardiovascular disease (CVD), there remains a large burden of residual disease due in part to persistent dyslipidemia characterized by elevated levels of triglyceride-rich lipoproteins (TRLs) and reduced levels of HDL. This form of dyslipidemia is increasing globally as a result of the rising prevalence of obesity and metabolic syndrome. Accumulating evidence suggests that impaired hepatic clearance of cholesterol-rich TRL remnants leads to their accumulation in arteries, promoting foam cell formation and inflammation. Low levels of HDL may associate with reduced cholesterol efflux from foam cells, aggravating atherosclerosis. While fibrates and fish oils reduce TRL, they have not been uniformly successful in reducing CVD, and there is a large unmet need for new approaches to reduce remnants and CVD. Rare genetic variants that lower triglyceride levels via activation of lipolysis and associate with reduced CVD suggest new approaches to treating dyslipidemia. Apolipoprotein C3 (APOC3) and angiopoietin-like 3 (ANGPTL3) have emerged as targets for inhibition by antibody, antisense, or RNAi approaches. Inhibition of either molecule lowers TRL but respectively raises or lowers HDL levels. Large clinical trials of such agents in patients with high CVD risk and elevated levels of TRL will be required to demonstrate efficacy of these approaches.
\end{abstract}

\section{Introduction}

Imagine a 60-year-old patient with metabolic syndrome (obesity, hypertension, insulin resistance, and dyslipidemia) who is taking a statin and has an LDL-cholesterol (LDL-C) level of $70 \mathrm{mg} / \mathrm{dL}$ but also has elevated triglycerides (TGs; $200 \mathrm{mg} / \mathrm{dL}$ ) and low HDL-cholesterol (HDL-C; $30 \mathrm{mg} / \mathrm{dL}$ ). What could be approaches to reduce atherosclerotic cardiovascular disease (CVD) risk in this patient? Approaches could first include further efforts to reduce LDL-C by maximizing the dose of potent statins, adding the cholesterol absorption inhibitor ezetimibe, or suppressing the LDL-R-regulating protein PCSK9 with monoclonal antibodies. While each of these treatments reduces CVD risk, they do not very consistently reduce TG levels and they still leave a substantial residue of CVD events (1-3). Treatment options for further lowering TGs might include fibrates or fish oils; however, the evidence for a beneficial effect of fibrates is not compelling (4), and while some fish oils may reduce CVD (5), the underlying mechanisms and impact remain uncertain. Although low HDL-C is associated with increased CVD risk, there are currently no effective drugs for targeting low HDL, and the whole idea of raising HDL-C has been called into question (6). Thus, beyond LDL-C, there are no optimal current treatment options to address dyslipidemia, as exemplified by this typical patient with metabolic syndrome.

Conflict of interest: ART is on the scientific advisory board and is a cofounder of Staten Biotechnology and has consulted for Amgen, Commonwealth Serum Laboratories, the Medicines Company, AstraZeneca, and Foresite Labs. IJG has received laboratory support and consulting fees from Arrowhead Pharmaceuticals and has consulted for Ionis Pharmaceuticals/Akcea Therapeutics.

Copyright: ( 2022 , Tall et al. This is an open access article published under the terms of the Creative Commons Attribution 4.0 International License.

Reference information: J Clin Invest. 2022;132(1):e148559.

https://doi.org/10.1172/JCl148559.
A major theme of this Review is that lowering levels of TG-rich lipoproteins (TRLs) by activation of lipolysis and enhanced hepatic clearance of cholesterol-rich TRL remnants is likely to be beneficial for CVD (Figure 1). Activation of lipolysis leads to reduction in the levels of atherogenic TRL as well as increased levels of HDL: both effects may reduce atherosclerosis. While diet, exercise, and weight loss can have an important role in lowering TGs and raising HDL-C (7-10), the emphasis here will be on treatments using novel technologies to target new pathways that have been uncovered through genetic studies.

\section{Trends in residual risk}

CVD, including myocardial infarction and stroke, is the leading cause of death in the United States and accounts for $28 \%$ of overall mortality (11). CVD risk factors include age, sex, hypertension, diabetes, smoking, body mass index, and increased levels of LDL-C or TGs or reduced HDL-C levels $(1,12)$. Current therapies for prevention of ischemic events focus on controlling risk factors, as well as suppressing thrombosis in at-risk individuals, and this approach has contributed to decades of improvement in CVD mortality (13). This trend has stalled in recent years, in part because of the rise of obesity, diabetes, and their associated dyslipidemias. The metabolic syndrome has become more prevalent in all sociodemographic groups in the United States, and now occurs in more than one-third of adults (14).

Both fasting and nonfasting TG levels associate with CVD risk. In a European population, $27 \%$ of adults had nonfasting TGs greater than $176 \mathrm{mg} / \mathrm{dL}$, a level that was associated with an approximately 1.9-fold increase in risk of CVD (15). In individuals with TGs greater than $580 \mathrm{mg} / \mathrm{dL}$, the risk was increased even further to 5.1-fold for myocardial infarction and 3.2-fold for 


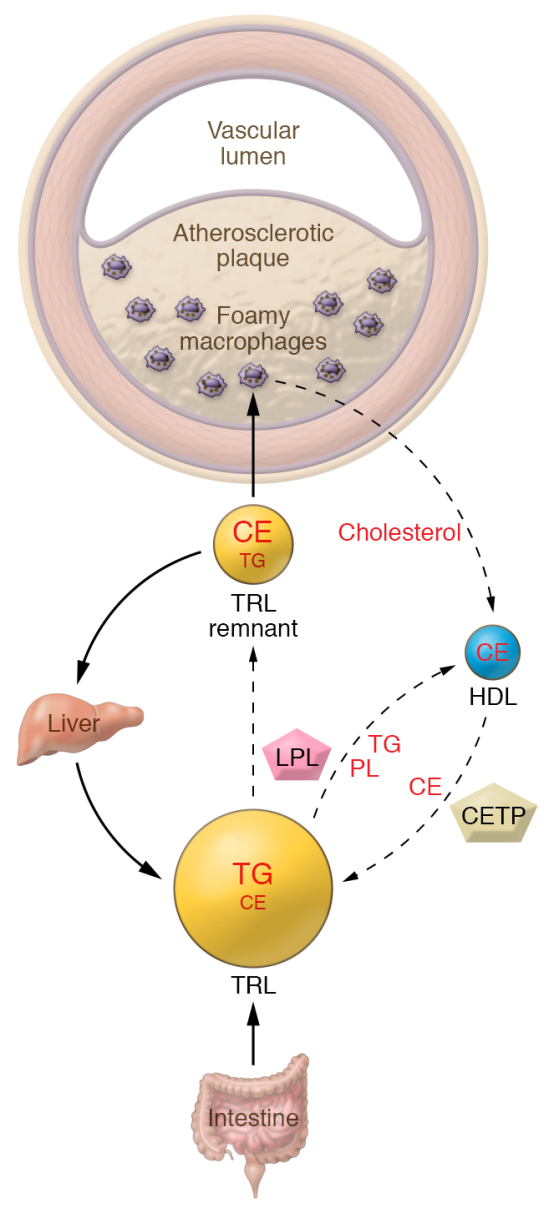

Figure 1. Lipoprotein modulation of atherosclerosis, beyond LDL. Circulating lipoproteins other than LDL modulate the development of atherosclerosis. Animal and human data show that cholesteryl ester-rich lipoproteins derived from the partial catabolism of TRLs, referred to as remnants, are taken up by macrophage foam cells in arteries, promoting development of atherosclerotic plaques. The catabolism of TRLs also mediates enrichment of HDL with phospholipids, increasing their ability to promote efflux of cholesterol from foam cells and thus ameliorating atherosclerosis. The interchange of lipids between HDL and TRL is mediated by cholesteryl ester transfer protein (CETP) and phospholipid transfer protein (not shown). CE, cholesteryl ester; LPL, lipoprotein lipase; PL, phospholipids.

ischemic stroke in comparison with subjects with low TG levels. The calculated remnant cholesterol level (total cholesterol minus LDL-C minus HDL-C) appears to provide comparable information on risk. Statins can moderately lower fasting and postprandial TG levels in hypertriglyceridemic patients (16), likely via enhanced hepatic LDL receptor-mediated (LDLR-mediated) remnant clearance. TG lowering is associated with reduced CVD risk in statin trials $(1,17)$, and elevated TG levels associate with residual risk in statin-treated patients, particularly in those with diabetes (18). Moreover, marked reductions in non-HDL cholesterol and to a lesser extent TGs associate with plaque regression as determined by intravascular ultrasound of coronary arteries (19). Several animal studies suggest that large minimally metabolized TRLs have reduced atherogenic potential compared with smaller TRL remnants or LDL. Such particles become the major circulating lipoproteins when cholesterol-fed rabbits are made diabetic with alloxan, but the particles penetrate the artery relatively poorly, and diabetic rabbits have less atherosclerosis than nondiabetic rabbits with smaller, more penetrant lipoproteins (20). Deletion of either lipoprotein lipase (LPL; ref. 21) or glycosylphosphatidylinositol-anchored HDL-binding protein 1 (GPIHBP1; ref. 22) causes severe hypertriglyceridemia, but mice only develop early atherosclerotic lesions at an advanced age. In mouse models of atherosclerosis regression, conditional knockout of LPL induced hypertriglyceridemia, reflecting increased nascent TRL but not VLDL-C or remnants, and did not affect atherosclerotic burden or morphology (23). Overall these observations suggest that while lowering TRL or more specifically remnant cholesterol levels is likely to reduce coronary atherosclerosis and CVD, many patients are inadequately treated by current medications, highlighting the need for new approaches to reduce TRL remnant levels.

\section{Insights from lipid trait genetics}

While observational studies have linked HDL-C and TGs to CVD risk (24), such studies have a limited role in elucidating the causal risk conferred by these lipid fractions because of confounding by correlated metabolic syndrome traits and artifacts introduced by differential variability of these analytes (25). Human genetic variation in lipid trait-modifying genes has provided key insights into mechanisms and therapeutic opportunities in atherosclerosis. Rare mutations increasing LDL-C are consistently associated with increased CVD risk proportionate to the level of LDL-C elevation, and the study of common variants in LDL-C-modifying genes recapitulates this observation $(26,27)$. In contrast, genetic associations of HDL-C and TGs with CVD risk based on rare variants do not unambiguously identify the culprit lipoprotein, in part because of concurrent effects on multiple lipoprotein traits. With the availability of large genome-wide association studies of lipid traits and CVD, common variants in HDL-C- and TG-modifying genes have been used to model the causal effects of changes in HDL-C and TGs on CVD through the Mendelian randomization approach (28). Since the genotype is randomly allocated in the population being studied, it can be inferred that the CVD effect derives from either the lipid trait effect or cosegregating pleiotropic effects of the genetic variant. Multiple variants can be simultaneously analyzed by meta-analysis to increase power and reduce bias conferred by isolated pleiotropic effects of single variants (29-31).

Early Mendelian randomization studies confirmed the role of LDL-C in CVD and suggested that levels of TGs, but not HDL-C, causally contribute to CVD risk $(30,32)$. Subsequently, the application of meta-analysis in Mendelian randomization, which accounted for differences in measurement error between variants, suggested causal effects of both HDL-C and TGs $(31,33)$. Further analyses using methods robust to pleiotropy, e.g., Egger regression, called into question the causal effects of HDL-C and TGs in CVD $(31,34)$. The TG effects on CVD were attenuated by adjustment for apolipoprotein B (APOB) levels, suggesting that TG levels are a biomarker for a variable summarized by APOB levels, e.g., the cholesterol content of TRL (35). Studies conducted in the Icelandic population also suggested that the deleterious effect of TG-raising genetic variants is accounted for by the non-HDL-C lipid fraction and mediated by the atherogenic effect of TRL-C $(36,37)$. 
A

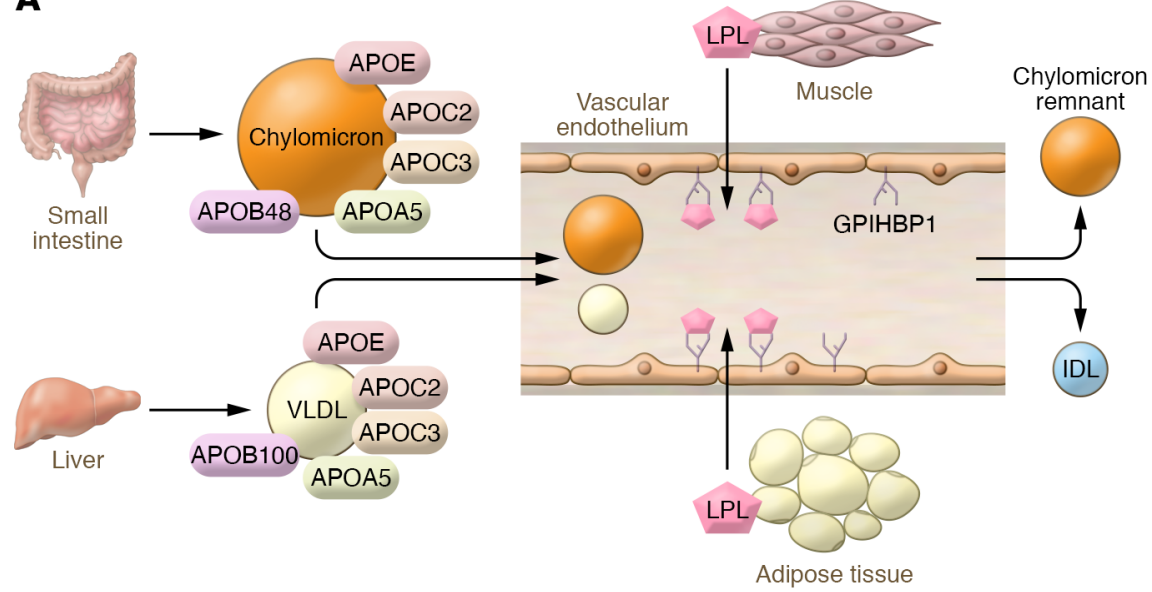

B

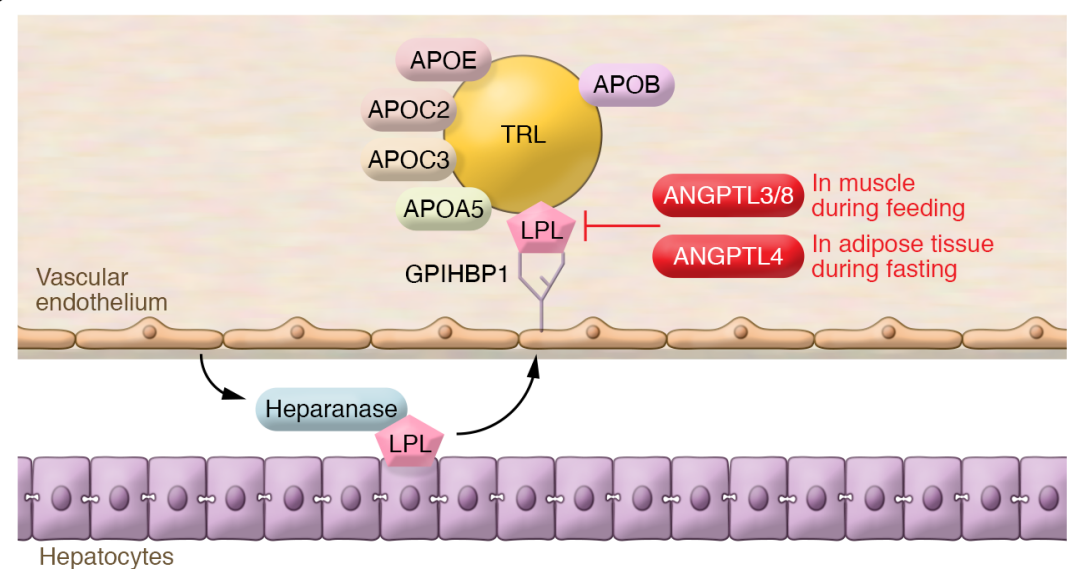

Figure 2. Lipolysis and TRL metabolism. (A) Lipolysis of circulating TRLs. Chylomicrons assembled in the small intestine and VLDL assembled in the liver contain proteins that control their intravascular metabolism. APOC2 is the activator of LPL. APOA5 also acts to enhance lipolysis, while APOC3 inhibits lipolysis. LPL is predominantly synthesized in adipose tissue, skeletal muscle, and heart. LPL transfers to the capillary lumen, where it associates with glycosylphosphatidylinositol-anchored HDL-binding protein 1 (GPIHBP1), releases free fatty acids from TRLs, and creates chylomicron remnants and intermediate-density lipoproteins (IDLs). (B) Lipolysis reaction. TRLs associate with LPL in the capillary lumen, a process thought to be assisted by APOA5. APOC2 activates LPL; APOC3 inhibits LPL. ANGPTLs also inhibit LPL. ANGPTL3, primarily produced in the liver, is most active in complex with ANGPTL8. ANGPTL4, though widely expressed, modulates LPL activity especially in adipose tissue.
Recently, our multivariable analysis using very large data sets and adjustment for widespread pleiotropic effects of lipid trait variants on metabolic syndrome traits revealed independent causal effects of HDL-C and TGs on CVD albeit of smaller magnitude than the well-known LDL-C effect (38). Our finding that LDL-C, HDL-C, and TGs are independently associated with coronary artery disease (CAD) by Mendelian randomization (38) builds on three recent reports showing independent Mendelian randomization associations of LDL-C, HDL-C, and TGs with abdominal aortic aneurysm $(34,39,40)$, suggesting that each of these factors is biologically active at the level of the arterial wall. Our study suggested that some, but not all, mechanisms of HDL-C raising and TG lowering are associated with protective CAD effects. These mechanism-specific effects may have been missed in prior studies because of both effect heterogeneity and statistical power limitations related to sample size, which were magnified by the intrinsic power limitations of pleiotropy-adjusted Mendelian randomization techniques (41), but are concordant with the results of other recent studies in large cohorts identifying a causal CAD effect for subsets of HDL-C variants (42-44). HDL-C-raising variants in genes such as LIPG (encoding endothelial lipase) that regulate HDL catabolism and may have downstream effects on macrophage cholesterol efflux had causal protective effects on CVD (38). LIPG activity leads to catabolism of HDL phospholipids and may cause the resulting phospholipid-poor HDL to be a poorer cholesterol acceptor $(45,46)$. The rs77960347
SNP in LIPG encoding the N396S mutation confers a large increase in HDL-C. In Mendelian randomization based on CAD data from 20,913 CAD cases and 95,407 controls, a null association of LIPG N396S with CAD was reported (32). In a subsequent CAD genomewide association study comprising 60,801 CAD cases and 123,504 controls (47), LIPG N396S was associated with a possible CAD effect with an odds ratio of $0.90(P=0.05)$. In our recent CAD meta-analysis of 122,733 CAD cases and 424,528 controls, LIPG N396S was associated with a CAD effect with an odds ratio of 0.90 (95\% CI $\left.0.86-0.95 ; P=8.8 \times 10^{-5}\right)(38)$. Consequently, univariable Mendelian randomization on this single SNP reveals a causal protective effect of increased HDL-C conferred by LIPG activity. In contrast, HDL-Craising variants in genes that regulate the cholesteryl ester content of HDL particles, such as LCAT (encoding lecithin-cholesterol acetyltransferase), actually increase CVD risk. Likewise, TG-raising variants in genes that regulate the catabolism of TRLs such as $L P L$ or ANGPTL4 increased CVD risk, whereas TG-raising variants in genes that regulate hepatic TG biosynthesis such as MLXIPL (encoding carbohydrate-responsive element-binding protein [ChREBP]) or FADS1 (encoding fatty acid desaturase 1) had no causal effect on CVD. Thus, while our meta-analysis adjusted for pleiotropy showed overall causal effects of TG and HDL-C levels on CAD, Mendelian randomization at loci with well-established direct effects on HDL-C and TG levels indicates locus- and mechanism-specific causal effects of these factors on CAD (38). 


\section{CARDIOVASCULAR DISEASES}

A

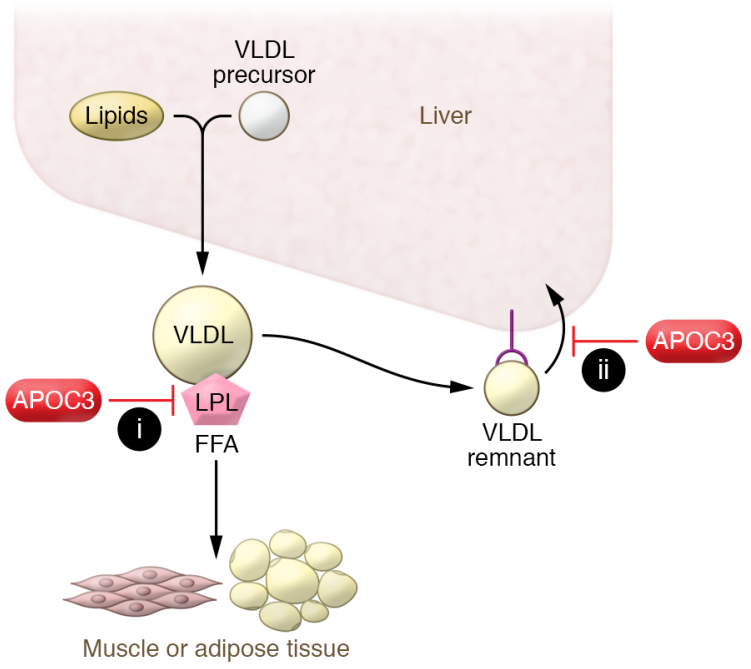

B

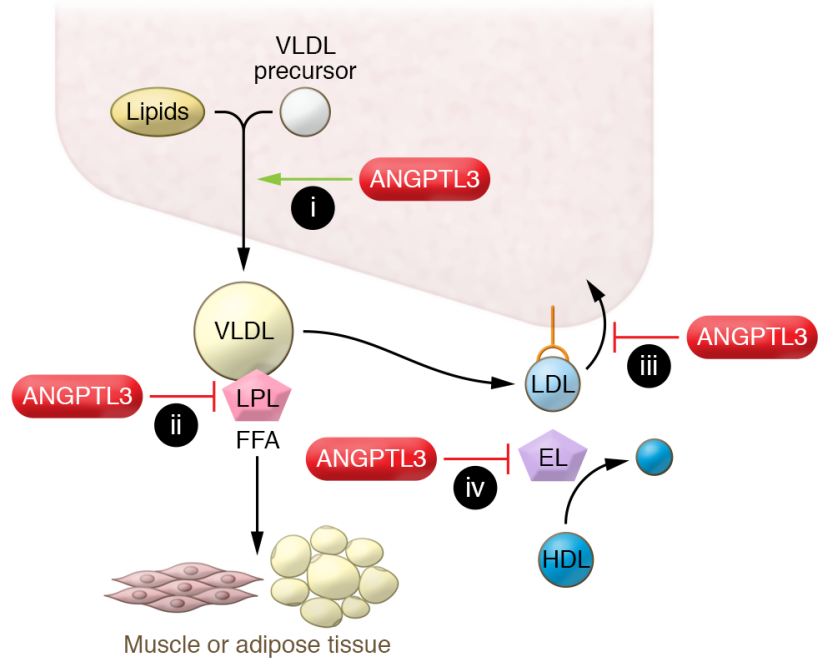

Figure 3. Two potential new therapies to reduce TRLs. (A) APOC3 depletion via knockdown in the liver or antibody inhibition in the circulation reduces circulating TC levels via two mechanisms: (i) removal of APOC3 releases its inhibition of LPL and increases intravascular lipolysis, and (ii) loss of APOC3 promotes uptake of TRL in the liver. (B) ANGPTL3 depletion reduces TRLs and LDL via (i) reduced liver TC secretion; (ii) increased intravascular lipolysis; and increased hepatic removal via either (iii) LDLR-dependent, non-endothelial lipase-dependent or (iv) non-LDLR-dependent, endothelial lipase-dependent processes. HDL levels decrease with ANGPTL3 loss as a result of activation of endothelial lipase. EL, endothelial lipase; FFA, free fatty acid.

\section{Recent developments in understanding of TRL metabolism}

TRLs transfer TGs from the diet or liver to tissues such as muscle and adipose for energy or fuel storage. Chylomicrons and VLDL require a lipolysis step to break the ester bond between fatty acids and glycerol (Figure 2A); luminal endothelial cell-associated LPL mediates this reaction. LPL is a member of a gene family that includes hepatic lipase and LIPG; the primary substrates of the latter enzymes are remnant lipoproteins and HDL. LPL binds to GPIHBP1 (48), which protects it from inactivation by angiopoietin-like 4 (ANGPTL4) (Figure $2 \mathrm{~B}$ and ref. 49). Activity of LPL is also modulated by fatty acids that inhibit its catalytic activity and release it from endothelial cells.

The protein cargo of circulating TRLs regulates their intravascular metabolism (Figure 2A). The APOC proteins are components of TRLs and HDL and transfer from HDL to TRL in the postprandial state (50). APOC2 is the obligate activator of LPL, and APOC2 deficiency leads to familial chylomicronemia syndrome. A recent study has shown that an APOC2 analog that also reduces APOC3 increases lipolysis and reduces circulating TG levels (51). LPL activity is inhibited by APOC3, perhaps because it shields TGs or blocks association of LPL and APOC2. APOC3 also inhibits remnant clearance (see below). APOA5 deficiency leads to hypertriglyceridemia, which may reflect its role in the efficient association of TRL with LPL or GPIHBP1 on endothelial cells (52), possibly because APOA5 interferes with an inhibitory effect of ANGPTL3/ANGPTL8 on this association (53).

ANGPTL3 and ANGPTL4 bind to the C-terminal region of LPL, disrupting its tertiary structure and canceling its activity in a tissue-specific fashion (Figure 2B and ref. 49). ANGPTL4 is induced in adipose tissue during fasting, inhibiting the lipolytic uptake of fatty acids (54). ANGPTL3 is primarily synthesized in the liver and associates with circulating ANGPTL8 to enhance its LPL-inhibitory actions in muscle, thus acting in an endocrine fashion $(55,56)$. ANGPTL8 is also synthesized in adipose tissue and acts in a juxtacrine or paracrine fashion to block the inhibitory effect of ANGPTL4 on adipose LPL through formation of ANGPTL4/ANGPTL8 complexes $(56,57)$. ANGPTL8 is induced in both liver and adipose tissue in the postprandial state, thus leading to increased uptake of fatty acids in adipose tissue and reduced uptake in muscle $(56,57)$. The complex posttranslational regulation of LPL activity by ANGPTLs therefore controls the tissue distribution of TG-derived fatty acids during feeding and fasting.

Tissue uptake of lipids from chylomicrons differs from that of lipids from VLDL in part because of differences in the movement of lipoprotein lipids across the endothelial cell barrier. This might reflect the local concentrations of fatty acids, which likely are much greater after lipolysis of chylomicrons versus VLDL. Although endothelial cells can internalize and degrade TRLs in lysosomes $(58,59)$, uptake of TRL TGs primarily occurs after LPL generation of nonesterified fatty acids (NEFAs). Studies of the fatty acid transporter CD36 in endothelial cells (60) implicate this receptor in fatty acid uptake when NEFA levels are low (61). Therefore, CD36 might be most important during fasting or perhaps VLDL hydrolysis; with higher NEFA levels, the receptor plays a less important role. Consistent with this, chylomicron-derived NEFAs and cholesteryl esters do not require CD36 for their movement into the heart $(62,63)$, and likely either other organs use other receptors or chylomicron-derived NEFAs cross the endothelial barrier via a paracellular route. In contrast, uptake of VLDL-derived lipids into the heart (62) and brown adipose tissue (64) is reduced by CD36 deficiency.

\section{Potential mechanisms of benefit from lowering of TRLs}

The cholesterol-rich remnants of chylomicrons or VLDL accumulate in the arterial intima, promoting macrophage foam cell formation and inflammatory changes in atherosclerotic plaques (Fig- 
ure 1). However, as with LDL, it is not completely clear what are the most important toxic molecules in TRL remnants; candidates include cholesterol, phospholipids or their oxidation products, fatty acids derived from lipolysis, and immunogenic APOB. The strongest evidence suggests an atherogenic role of TRL remnant cholesterol (15). Cholesterol-rich remnant particles carry a bigger load of cholesterol, are more effective at inducing macrophage foam cells than LDL, and do not need to be modified by oxidation in order to be taken up by macrophages (18). Free fatty acids liberated during lipolysis of TRL on the endothelial surface also can exert proinflammatory effects on endothelial cells and macrophages $(65,66)$.

Despite the correlation of atherosclerosis risk with VLDL or LDL cholesterol, recent immune cell profiling of atherosclerotic plaques by single-cell RNA sequencing studies has introduced a conundrum: Trem2-high cholesteryl ester-laden foam cells have lower expression of inflammatory genes compared with other populations of macrophages in plaques (67). Glass and colleagues have shown that cholesterol-laden foam cells suppress inflammatory pathways as a result of accumulation of desmosterol, a ligand of liver X receptor (LXR) (68); LXR activation reduces expression of inflammatory genes by cis-repression and other mechanisms (69). Recent evidence also highlights the atherogenic role of lipoprotein-derived oxidized phospholipids (70). Together these studies suggest that oxidative or other modifications of remnant particles or LDL that produce danger-associated molecular patterns to activate Toll-like receptor signaling may be more important in inducing inflammatory responses than macrophage cholesterol loading per se. However, excessive cholesterol loading that is not compensated by cholesterol esterification or cholesterol efflux may lead to ER stress and cell death (71), as well as to activation of the NLRP3 inflammasome and formation of neutrophil extracellular traps $(72,73)$, contributing to the formation of unstable plaques, plaque erosion, and thrombosis (74).

\section{Dyslipidemia and vascular inflammation}

Recent human CVD outcome trials using IL-1 $\beta$ antibodies (75) or colchicine (76) have demonstrated reduced CVD. This underlines the role of inflammation in the clinical complications of atherosclerosis and opens a new vista on antiinflammatory therapies as potential treatments for atherosclerotic CVD. However, these studies should not be viewed in isolation, because, as indicated above, the inflammatory component of atherosclerosis is intimately connected to the dyslipidemia that promotes the accumulation of atherogenic lipoproteins in the artery wall. Accordingly, elevated levels of remnant cholesterol are associated with higher CRP levels, a biomarker of inflammation (77). Moreover, dyslipidemia activates hematopoiesis and myelopoiesis, promoting formation of increased numbers of inflammatory monocytes and neutrophils that are poised to enter the artery wall and promote atherothrombosis (78-81). Thus, dyslipidemia contributes to the inflammatory risk of atherosclerosis. In some patients, vigorous control of dyslipidemia may adequately reduce inflammatory risk, obviating the need for potentially immunosuppressive antiinflammatory treatments. However, in others genetic inflammatory risk may be increased (e.g., as in clonal hematopoiesis of indeterminate potential; refs. 82-84) and control of dyslipidemia may need to be buttressed with antiinflammatory therapies. A recent study showed that antiinflammatory therapies that lowered CVD risk were associated with reductions in the neutrophil/lymphocyte ratio, a global marker of inflammation; however, lipid lowering with statins or PCSK9 inhibitors did not affect this ratio (85). Thus, targeting antiinflammatory therapies to susceptible populations identified by genetic risk factors or by appropriate biomarkers may be an important addition to controlling dyslipidemia.

\section{Emerging targets in dyslipidemia based on genetic studies}

APOC3. It has long been known that plasma APOC3 concentrations correlate with TG levels (86), are elevated in patients with diabetes, and associate with increased coronary atherosclerosis and CVD risk $(87,88)$. The causal role of APOC3 in increased TG levels was first shown by overexpression of APOC 3 in transgenic mice (89), and the pro-atherogenic role of APOC3 was demonstrated in APOC3-transgenic, LDLR-deficient mice (90). Animal and cell studies suggest that APOC3 deficiency has two main TG-lowering effects: activation of LPL-mediated lipolysis and promotion of the hepatic clearance of TRL remnants (Figure 3A). APOC3 inhibits the hepatic clearance of TRL remnants via the LDLR and LDLR-related protein (LRP) (91). The effects of APOC3 inhibition on hepatocyte clearance of TRL remnants are not seen in the absence of APOE and likely reflect reduced masking of APOE by APOC3 rather than reduced displacement of APOE (92). However, APOC3 deficiency or inhibition lowers TG levels in the absence of APOE likely as result of increased LPL activity and uptake of fatty acids in adipose tissue (92).

The translational importance of these studies in animals and cells was illuminated by human genetic findings. Shuldiner and colleagues (93) showed that loss-of-function APOC3 variants in the Amish associated with reduced TG levels and decreased coronary atherosclerosis. These findings were extended in large population studies in the National Heart, Lung, and Blood Institute Exome Sequencing Project (94) and in two other European population studies (95). Four different APOC3 variants were found: three nonsense mutations and one missense mutation with an overall frequency of 1 in 150 . In heterozygotes, there was a $39 \%$ lowering of TGs, a $46 \%$ lowering of APOC3 levels, a $25 \%$ increase in HDL-C, and a $40 \%$ reduction in CVD risk (94). In a smaller study in the Icelandic population, the most common APOC3 loss-of-function variant did not associate significantly with CVD, consistent with a more modest effect size (36). Homozygous APOC3 deficiency is associated with markedly reduced fasting and postprandial TG levels and appears not to have adverse health effects (96).

The impact of heterozygous loss-of-function APOC3 mutations on LDL-C is minimal (-4\%) (95), suggesting the importance of lowered remnant cholesterol and possibly increased HDL in the beneficial effects of APOC3 deficiency. Lipoprotein turnover studies in small groups of subjects with homozygous (93) or heterozygous (97) deficiencies of APOC3 showed increased fractional clearance of VLDL TG and APOB, and increased conversion of VLDL to LDL without clearly increased hepatic clearance of remnants. However, in large population studies, APOC3 deficiency was associated with reduced VLDL and non-HDL cholesterol levels that appeared to be largely responsible for the CVD benefit (98). APOC3 might have 
additional atherogenic effects. LDL enriched with APOC3 associates strongly with CVD risk (99), perhaps because APOC3 increases LDL affinity for proteoglycans (100), and as mentioned above, APOC3 may increase vascular inflammation.

An antisense oligonucleotide (ASO) to APOC3 (volanesorsen) very effectively lowered TG levels in patients with familial chylomicronemia syndrome (FCS), including those with LPL-null mutations (101). Volanesorsen lowered TG levels in patients with moderately severe hypertriglyceridemia (mean TGs $=580 \mathrm{mg}$ / $\mathrm{dL}$ ), but also resulted in significant LDL-C elevations and no overall change in non-HDL cholesterol or APOB levels (102). Volanesorsen treatment was subsequently found to induce clinically significant thrombocytopenia in a few patients with FCS (103). The use of hepatocyte-targeted ASOs to lower APOC3 is still under investigation as a therapeutic for FCS and recurrent pancreatitis (101). RNAi approaches have recently been introduced to reduce hepatic PCSK9, producing sustained LDL lowering after only two doses (104). RNAi lowering of APOC3 has also been reported with potent TG and APOC3 lowering, increased HDL-C, and prolonged effects of more than 10 weeks (105). Monoclonal antibody approaches to APOC3 lowering have also been described (106). A potential advantage of the antibody approach is their ability to inhibit APOC3 produced both in the liver and in the intestine, while a challenge is that APOC3 is an abundant plasma protein.

ANGPTL3. ANGPTL3 deficiency causes pan-hypolipidemia (107) and reduces CVD risk (108). Relative to individuals with functional ANGPTL3, subjects with various rare heterozygous loss-of-function mutations had mean TGs decreased by $27 \%$, LDL-C by $9 \%$, and HDL-C by $4 \%$ with an odds ratio for CVD of 0.61. ANGPTL3 antibodies (evinacumab) and ASOs (vupanorsen) (109) are being developed as potential treatments of CVD. ANGPTL3 antibodies lower LDL-C independently from LDLR activity, and, notably, evinacumab lowers LDL-C by about $50 \%$ in familial hypercholesterolemia homozygotes (110). In addition to inhibiting LPL, ANGPTL3 also inhibits activity of LIPG, and this accounts for the HDL-lowering effect of ANGPTL3 inhibition $(111,112)$. Two recent studies $(113,114)$ have shown that in the absence of LDLRs, VLDL/LDL-lowering effects of ANGPTL3 inhibition are mediated through increased LIPG activity. However, ANGPTL3 inhibition does lower LDL-C in $\mathrm{Lipg}^{-/-}$mice lacking LIPG but with functioning LDLR (113). Thus, ANGPTL3 reduction appears to increase two pathways of LDL-C reduction, one via the LDLR independent of LIPG and a second requiring LIPG but not the LDLR (Figure 3B).

An LIPG reduced-function variant that increases HDL-C and to a much lesser extent non-HDL cholesterol is associated with reduced CVD (38). $\mathrm{Lipg}^{-/-}$mouse models have shown either reduced (115) or unchanged atherosclerosis (116).Together these studies suggest that in the presence of functioning LDLR the benefit of ANGPTL3 inhibition may be attenuated by increased LIPG activity as a result of reduced HDL-C and reduced cholesterol efflux capacity (117). While evinacumab is being developed for treatment of familial hypercholesterolemia, the potential for wider application in dyslipidemia could be limited by HDL-C-lowering effects. A beneficial effect of ANGPTL3 inhibition on insulin resistance and fatty liver has been suggested $(109,118)$; however, liver-targeted antisense inhibition of ANGPTL3 with vupanorsen in subjects with fasting hypertriglyceridemia and increased liver fat, while lowering TG levels by about $60 \%$, did not result in reduced steatosis or improved glycemic parameters (119).

ANGPTL4. Reduced-function variants of ANGPTL4 have been associated with lower TG levels, increased HDL-C, and reduced CVD risk (120). This suggested that, like targeting of ANGTPL3, targeting of ANGPTL4 could lower TGs and CVD risk. However, in mice, homozygous Angptl4 deficiency resulted in lipogranulomatous lesions of the intestines and their draining lymphatics, probably reflecting premature lipolysis of chylomicrons and macrophage inflammation $(121,122)$. These adverse effects, although not reported in humans, appear to have impeded the clinical development of ANGPTL4 inhibitors. However, deletion of ANGPTL4 in hepatocytes resulted in reduced TG levels and protected against diet-induced obesity, glucose intolerance, liver steatosis, and atherosclerosis, without the previously described complications of whole-body Angptl4 deficiency (123).

Fish oils (n-3 long-chain polyunsaturated fatty acids). Marine oils lower plasma TG levels in humans and have been extensively assessed for potential CVD benefit. The TG-lowering mechanism of $n-3$ fatty acids is related to decreased hepatic VLDL production (124). Four grams per day of icosapent ethyl (Vascepa, an ester of eicosapentaenoic acid [EPA]) substantially reduced TG levels and CVD events in statin-treated patients with elevated TGs (135 to 499 $\mathrm{mg} / \mathrm{dL}$ ) in the REDUCE-IT trial (5); this is reminiscent of the results of an earlier open-label trial using $1.8 \mathrm{~g} / \mathrm{d}$ EPA in hypercholesterolemic Japanese patients $(125,126)$. TG lowering is unlikely to be the full explanation for the CVD benefit of icosapent ethyl, as benefits were similar irrespective of the degree of TG lowering in subjects receiving icosapent ethyl. In contrast, a lower dose of $1 \mathrm{~g}$ combined EPA/docosahexaenoic acid (DHA) failed to show a CVD benefit in diabetics (127), and $4 \mathrm{~g} / \mathrm{d}$ of a mixture of EPA/DHA versus corn oil failed to show a benefit in statin-treated subjects with high CVD risk in the STRENGTH trial (128). The apparent CVD benefit of icosapent ethyl may be related to the dose of EPA, differences between EPA and DHA, the targeting of a susceptible population with elevated TGs over $150 \mathrm{mg} / \mathrm{dL}$, and the long duration of the trial (5 years). However, concerns have been raised regarding the use of mineral oil as a control, which could potentially have decreased statin absorption, as control subjects experienced an increase in LDL-C and CRP (129). Modeling of the predicted CVD effects of observed plasma lipid and CRP changes resulting from administration of mineral oil or EPA suggests that a considerable part of the apparent CVD benefit in REDUCE-IT was related to adverse effects of mineral oil in the control group, while about $13 \%$ of the CVD benefit was related to effects of EPA or mineral oil not mediated by lipid and CRP changes (124, 130-132). Increased formation of inflammation resolution mediators could be part of the benefit derived from EPA; failed inflammation resolution can impair regression of atherosclerosis (133). Further studies on fish oils may help to provide a clearer understanding of the mechanisms and magnitude of their CVD benefit.

\section{Challenges in the development of new}

\section{TC-lowering agents}

In contrast to LDL lowering, it is unlikely that all modes of TG lowering will have similar impact on CVD. Our Mendelian randomization studies suggest that genetic variants that cause acti- 
vation of lipolysis may have beneficial effects on CVD, while some variants that decrease VLDL production do not associate with reduced CVD (38). Decreasing VLDL production, e.g., by APOB ASO treatment, may also lead to nonalcoholic fatty liver disease (134). Fibrates lower TG levels by decreasing APOC3 and increasing lipolysis; however, fibrates have pleiotropic effects, and the lack of clear-cut benefits has impeded their acceptance. A new class of fibrates is being evaluated in patients with high TGs and low HDL (135).

The amount of TG lowering in clinical trials that is likely to produce benefit is unclear. As with LDL-C, the reduction in CVD risk from TG or remnant-cholesterol lowering appears to be proportional to the absolute reduction in TRL levels, and thus for a given percentage reduction in TGs, the impact will be larger when baseline TG levels are higher $(17,136)$. This emphasizes the importance in clinical trials of targeting populations with substantial elevation in TG levels at baseline, as was done in the more successful trials of fibrates $(4,136)$ and of icosapent ethyl $(135)$.

\section{Potential benefits of increasing reverse cholesterol transport}

Activation of lipolysis of TRL leads to an increase in HDL levels as a result of the transfer of surface phospholipids and APOA1 from TRL into HDL as well as reduced cholesteryl ester transfer protein-mediated (CETP-mediated) exchange of TRL TG for HDL cholesteryl ester (Figure 1). The enrichment of HDL with phospholipids and APOA1 is likely to increase its ability to promote efflux of cholesterol from macrophages and endothelial cells in atheromatous plaques via ABCA1 and ABCG1 transporterdependent and other cholesterol efflux pathways $(137,138)$. A number of studies indicate that the ability of HDL to promote efflux of cholesterol from macrophages is inversely correlated with CVD risk (139). Although cholesterol efflux is correlated with HDL-C levels, multivariate analysis has shown a strong effect of cholesterol efflux on CVD independent of HDL-C levels. Higher levels of cholesterol efflux capacity may be related to an increased content of phospholipids in HDL (140), which could also explain the apparent CVD benefit of the LIPG N396S reduced-function variant (38). The cholesterol mass efflux capacity of HDL shows an inverse relationship to coronary artery disease but not to thrombotic stroke (141) or peripheral artery disease (142), indicating a differential effect of HDL-mediated macrophage cholesterol efflux in different vascular beds. A recent study has shown that the ability of HDL to suppress inflammatory responses in cultured endothelial cells is inversely correlated with incident CVD and apparently independent of macrophage cholesterol efflux capacity (143).

LXR activators appear to be ideal drugs for increasing reverse cholesterol transport and suppressing plaque inflammation (144). They are potently and consistently anti-atherogenic in animal models. However, clinical development in humans was halted because of adverse effects on hepatic steatosis and liver function tests and an increase in LDL-C levels that likely reflects a mechanism-related suppression of hepatic $\operatorname{LDLR}(145,146)$. As an alternative, plaque macrophage-targeted nanoparticles containing LXR activators might represent an attractive option for LXR therapeutics (147).

\section{HDL-directed therapeutics}

The results of clinical trials of agents that raise HDL-C have been disappointing (6). In particular, CETP inhibitors markedly increased HDL-C levels and moderately reduced LDL-C levels. While the largest and longest trial of CETP inhibition using anacetrapib showed a highly significant 9\% reduction in CVD endpoints (148), anacetrapib has not been marketed for CVD, probably owing to the moderate effect size and the long-term accumulation of the drug in adipose tissue. Moreover, this CVD reduction might primarily reflect the LDL-C reduction that occurred (149). HDL infusions and overexpression of APOA1 in animal models consistently reduce atherosclerosis (150-152), suggesting a direct anti-atherogenic action. This has led to clinical trials of infused reconstituted HDL (rHDL) particles consisting of phospholipids and APOA1. In animals, such preparations have anti-atherogenic (153) and antiinflammatory effects (154). Although proinflammatory effects of such preparations have been described, these largely occurred under conditions of extreme cholesterol depletion as a result of an ER stress response and do not occur in atherosclerotic plaques (154).

Infusions of early formulations of rHDL such as CSL-111 improved the characteristics of coronary artery plaques in imaging studies but also caused significant elevation of liver function tests (155). A new formulation of rHDL, CSL-112, containing reduced phospholipids relative to APOA1, produced lower levels of cholesterol efflux but did not induce elevations in alanine transaminase (a potential biomarker of liver injury) when infused into mice or humans (156-158). Four infusions of CSL-112 are currently being assessed as a treatment to prevent recurrent CVD in patients with severe coronary disease. Although this study represents a direct test of the anti-atherogenicity of HDL in humans, it remains to be seen whether the dose and duration of rHDL treatment will be sufficient to reduce CVD.

\section{Summary and perspective}

Goldstein et al. reported in this journal more than 40 years ago that hypertriglyceridemia was commonly found in patients with coronary artery disease (159). Zilversmit proposed that cholesterol-rich remnants of TRL might be atherogenic (160), and over the last 40 years epidemiological and genetic evidence has accrued to support the hypothesis that remnants increase CVD risk, while reduction of remnant levels by statins or other treatments may be beneficial. However, we still are uncertain as to the optimal lipid-modifying therapies for targeting TGs in the 60-year-old metabolic syndrome patient discussed in the introduction. There is hope that a new class of therapeutics based on rare mutations affecting TG levels will activate lipolysis, promote remnant clearance, and reduce CVD. To determine whether these new therapies reduce CVD will require large clinical outcome studies. That any CVD benefit derives directly from lowering TG or remnants is unlikely to be revealed because, like fibrates and fish oils before them, these newer TG-reducing medicines will have multiple effects on lipoproteins and other atherosclerosis risk factors. Nonetheless, while as a research community we continue to work toward a clearer understanding of the complicated biology associated with circulating lipoproteins and CVD, based on new approaches to lowering TRL and increasing HDL-C, clinical medicine will likely advance toward improved patient outcomes. 


\section{Acknowledgments}

This work was supported by NIH grants HL107653 (to ART) and HL45095 and HL151328 (to IJG).
Address correspondence to: Alan Tall, Columbia University Medical Center, 630 W. 168th St., New York, New York 10032, USA. Email: art1@cumc.columbia.edu.
1. Vallejo-Vaz AJ, et al. Triglyceride-rich lipoprotein cholesterol and risk of cardiovascular events among patients receiving statin therapy in the TNT trial. Circulation. 2018;138(8):770-781.

2. Cannon CP, et al. Ezetimibe added to statin therapy after acute coronary syndromes. $N$ Engl J Med. 2015;372(25):2387-2397.

3. Sabatine MS, et al. Evolocumab and clinical outcomes in patients with cardiovascular disease. N Engl J Med. 2017;376(18):1713-1722.

4. Jun $\mathrm{M}$, et al. Effects of fibrates on cardiovascular outcomes: a systematic review and meta-analysis. Lancet. 2010;375(9729):1875-1884.

5. Bhatt DL, et al. Cardiovascular risk reduction with icosapent ethyl for hypertriglyceridemia. N EnglJ Med. 2019;380(1):11-22.

6. Tall AR. HDL in morbidity and mortality: a $40+$ year perspective. Clin Chem. 2021;67(1):19-23.

7. Kraus WE, et al. Effects of the amount and intensity of exercise on plasma lipoproteins. $N$ EnglJ Med.2002;347(19):1483-1492.

8. Tall AR. Exercise to reduce cardiovascular risk - how much is enough? N Engl JMed. 2002;347(19):1522-1524.

9. Chan DC, et al. Effect of weight loss on markers of triglyceride-rich lipoprotein metabolism in the metabolic syndrome. Eur JClin Invest. 2008;38(10):743-751.

10. Miller M, et al. Triglycerides and cardiovascular disease: a scientific statement from the American Heart Association. Circulation. 2011;123(20):2292-2333.

11. Heron M. Deaths: leading causes for 2015. Natl Vital Stat Rep. 2017;66(5):1-76.

12. Herrington $\mathrm{W}$, et al. Epidemiology of atherosclerosis and the potential to reduce the global burden of atherothrombotic disease. Circ Res. 2016;118(4):535-546.

13. Virani SS, et al. Heart disease and stroke statistics-2021 update: a report from the American Heart Association. Circulation. 2021;143(8):e254-e743.

14. Moore JX, et al. Metabolic syndrome prevalence by race/ethnicity and sex in the United States, National Health and Nutrition Examination Survey, 1988-2012. Prev Chronic Dis. 2017;14:E24.

15. Nordestgaard BG. Triglyceride-rich lipoproteins and atherosclerotic cardiovascular disease: new insights from epidemiology, genetics, and biology. Circ Res. 2016;118(4):547-563.

16. Le NA, et al. Lipid and apolipoprotein levels and distribution in patients with hypertriglyceridemia: effect of triglyceride reductions with atorvastatin. Metabolism. 2000;49(2):167-177.

17. Marston NA, et al. Association between triglyceride lowering and reduction of cardiovascular risk across multiple lipid-lowering therapeutic classes: a systematic review and meta-regression analysis of randomized controlled trials. Circulation. 2019;140(16):1308-1317.

18. Chait A, et al. Remnants of the triglyceride-rich lipoproteins, diabetes, and cardiovascular disease. Diabetes. 2020;69(4):508-516.
19. Puri R, et al. Non-HDL cholesterol and triglycerides: implications for coronary atheroma progression and clinical events. Arterioscler Thromb Vasc Biol. 2016;36(11):2220-2228.

20. Nordestgaard BG, et al. Reduced atherogenesis in cholesterol-fed diabetic rabbits. Giant lipoproteins do not enter the arterial wall. Arteriosclerosis. 1988;8(4):421-428.

21. Zhang X, et al. Spontaneous atherosclerosis in aged lipoprotein lipase-deficient mice with severe hypertriglyceridemia on a normal chow diet. Circ Res. 2008;102(2):250-256.

22. Weinstein MM, et al. Chylomicronemia elicits atherosclerosis in mice-brief report. Arterioscler Thromb Vasc Biol. 2010;30(1):20-23.

23. Josefs $\mathrm{T}$, et al. Atherosclerosis regression and cholesterol efflux in hypertriglyceridemic mice. Circ Res. 2021;128(6):690-705.

24. Emerging Risk Factors Collaboration, et al. Major lipids, apolipoproteins, and risk of vascular disease. JAMA. 2009;302(18):1993-2000.

25. Davey Smith G, Phillips AN. Correlation without a cause: an epidemiological odyssey. Int JEpidemiol. 2020;49(1):4-14.

26. Linsel-Nitschke $P$, et al. Lifelong reduction of LDL-cholesterol related to a common variant in the LDL-receptor gene decreases the risk of coronary artery disease-a Mendelian Randomisation study. PLoS One. 2008;3(8):e2986.

27. Ference BA, et al. Variation in PCSK9 and HMGCR and risk of cardiovascular disease and diabetes. N Engl J Med. 2016;375(22):2144-2153.

28. Burgess S, et al. Use of Mendelian randomisation to assess potential benefit of clinical intervention. BMJ. 2012;345:e7325.

29. Palmer TM, et al. Using multiple genetic variants as instrumental variables for modifiable risk factors. Stat Methods Med Res. 2012;21(3):223-242.

30. Do R, et al. Common variants associated with plasma triglycerides and risk for coronary artery disease. Nat Genet. 2013;45(11):1345-1352.

31. White J, et al. Association of lipid fractions with risks for coronary artery disease and diabetes. JAMA Cardiol. 2016;1(6):692-699.

32. Voight BF, et al. Plasma HDL cholesterol and risk of myocardial infarction: a mendelian randomisation study. Lancet. 2012;380(9841):572-580.

33. Burgess S, et al. Using multivariable Mendelian randomization to disentangle the causal effects of lipid fractions. PLoS One. 2014;9(10):e108891.

34. Allara E, et al. Genetic determinants of lipids and cardiovascular disease outcomes: a wide-angled mendelian randomization investigation. Circ Genom Precis Med. 2019;12(12):e002711.

35. Holmes MV, Davey Smith G. Challenges in interpreting multivariable mendelian randomization: might "good cholesterol" be good after all? Am J Kidney Dis. 2018;71(2):149-153.

36. Helgadottir A, et al. Variants with large effects on blood lipids and the role of cholesterol and triglycerides in coronary disease. Nat Genet. 2016;48(6):634-639.

37. Bjornsson E, et al. Association of genetically predicted lipid levels with the extent of coronary atherosclerosis in Icelandic adults. JAMA Cardiol. 2020;5(1):13-20.

38. Thomas DG, et al. Lipid and metabolic syndrome traits in coronary artery disease: a Mendelian randomization study [published online February 6, 2021]. J Lipid Res. https://doi.org/10.1194/jlr. p120001000.

39. Klarin D, et al. Genetics of blood lipids among 300,000 multi-ethnic participants of the Million Veteran Program. Nat Genet. 2018;50(11):1514-1523.

40. Harrison SC, et al. Genetic association of lipids and lipid drug targets with abdominal aortic aneurysm: a meta-analysis. JAMA Cardiol. 2018;3(1):26-33.

41. Bowden J, et al. Mendelian randomization with invalid instruments: effect estimation and bias detection through Egger regression. Int J Epidemiol. 2015;44(2):512-525.

42. Zhao Q, et al. Powerful three-sample genomewide design and robust statistical inference in summary-data Mendelian randomization. Int $J$ Epidemiol. 2019;48(5):1478-1492.

43. Cheng Q, et al. MR-LDP: a two-sample Mendelian randomization for GWAS summary statistics accounting for linkage disequilibrium and horizontal pleiotropy. NAR Genom Bioinform. 2020;2(2):lqaa028.

44. Burgess S, et al. A robust and efficient method for Mendelian randomization with hundreds of genetic variants. Nat Commun. 2020;11(1):376.

45. Maugeais C, et al. Dose-dependent acceleration of high-density lipoprotein catabolism by endothelia lipase. Circulation. 2003;108(17):2121-2126.

46. Agarwala AP, et al. High-density lipoprotein (HDL) phospholipid content and cholesterol efflux capacity are reduced in patients with very high HDL cholesterol and coronary disease. Arterioscler Thromb Vasc Biol. 2015;35(6):1515-1519.

47. Nikpay M, et al. A comprehensive 1,000 Genomes-based genome-wide association meta-analysis of coronary artery disease. Nat Genet. 2015;47(10):1121-1130.

48. Beigneux AP, et al. Glycosylphosphatidylinositolanchored high-density lipoprotein-binding protein 1 plays a critical role in the lipolytic processing of chylomicrons. Cell Metab. 2007;5(4):279-291.

49. Kristensen KK, et al. Unfolding of monomeric lipoprotein lipase by ANGPTL4: insight into the regulation of plasma triglyceride metabolism. Proc Natl Acad Sci U S A. 2020;117(8):4337-4346.

50. Havel RJ, et al. Interchange of apolipoproteins between chylomicrons and high density lipoproteins during alimentary lipemia in man. JClin Invest. 1973;52(1):32-38.

51. Wolska A, et al. A dual apolipoprotein C-II mimetic-apolipoprotein C-III antagonist peptide lowers plasma triglycerides. Sci Transl Med. 2020;12(528):eaaw7905.

52. Gin P, et al. Binding preferences for GPIHBP1, a glycosylphosphatidylinositol-anchored protein 
of capillary endothelial cells. Arterioscler Thromb Vasc Biol. 2011;31(1):176-182.

53. Chen YQ, et al. ApoA5 lowers triglyceride levels via suppression of ANGPTL3/8-mediated LPL inhibition. J Lipid Res. 2021;62:100068.

54. Ruppert PMM, et al. Fasting induces ANGPTL4 and reduces LPL activity in human adipose tissue. Mol Metab. 2020;40:101033.

55. Haller JF, et al. ANGPTL8 requires ANGPTL3 to inhibit lipoprotein lipase and plasma triglyceride clearance. J Lipid Res. 2017;58(6):1166-1173.

56. Oldoni F, et al. ANGPTL8 has both endocrine and autocrine effects on substrate utilization. JCI Insight. 2020;5(17):e138777.

57. Chen YQ, et al. Angiopoietin-like protein 8 differentially regulates ANGPTL3 and ANGPTL4 during postprandial partitioning of fatty acids. J Lipid Res. 2020;61(8):1203-1220.

58. Fischer AW, et al. Lysosomal lipoprotein processing in endothelial cells stimulates adipose tissue thermogenic adaptation. Cell Metab. 2021;33(3):547-564.

59. Abumrad NA, et al. Endothelial cell receptors in tissue lipid uptake and metabolism. Circ Res. 2021;128(3):433-450.

60. Son NH, et al. Endothelial cell CD36 optimizes tissue fatty acid uptake. JClin Invest. 2018;128(10):4329-4342.

61. Febbraio $\mathrm{M}$, et al. A null mutation in murine CD36 reveals an important role in fatty acid and lipoprotein metabolism. J Biol Chem. 1999;274(27):19055-19062.

62. Bharadwaj KG, et al. Chylomicron- and VLDL-derived lipids enter the heart through different pathways: in vivo evidence for receptorand non-receptor-mediated fatty acid uptake. J Biol Chem. 2010;285(49):37976-37986.

63. $\mathrm{He} \mathrm{C}$, et al. NanoSIMS analysis of intravascular lipolysis and lipid movement across capillaries and into cardiomyocytes. Cell Metab. 2018;27(5):1055-1066

64. Bartelt A, et al. Brown adipose tissue activity controls triglyceride clearance. Nat Med. 2011;17(2):200-205.

65. Schwartz EA, Reaven PD. Lipolysis of triglyceride-rich lipoproteins, vascular inflammation, and atherosclerosis. Biochim Biophys Acta. 2012;1821(5):858-866.

66. Ting HJ, et al. Triglyceride-rich lipoproteins prime aortic endothelium for an enhanced inflammatory response to tumor necrosis factor-alpha. Circ Res. 2007;100(3):381-390.

67. Kim K, et al. Transcriptome analysis reveals nonfoamy rather than foamy plaque macrophages are proinflammatory in atherosclerotic murine models. Circ Res. 2018;123(10):1127-1142.

68. Spann NJ, et al. Regulated accumulation of desmosterol integrates macrophage lipid metabolism and inflammatory responses. Cell. 2012;151(1):138-152.

69. Thomas DG, et al. LXR suppresses inflammatory gene expression and neutrophil migration through cis-repression and cholesterol efflux. Cell Rep. 2018;25(13):3774-3785.

70. Que X, et al. Oxidized phospholipids are proinflammatory and proatherogenic in hypercholesterolaemic mice. Nature. 2018;558(7709):301-306.

71. Scull CM, Tabas I. Mechanisms of ER stress- induced apoptosis in atherosclerosis. Arterioscler Thromb Vasc Biol. 2011;31(12):2792-2797.

72. Westerterp M, et al. Cholesterol efflux pathways suppress inflammasome activation, NETosis, and atherogenesis. Circulation. 2018;138(9):898-912.

73. Duewell P, et al. NLRP3 inflammasomes are required for atherogenesis and activated by cholesterol crystals. Nature. 2010;464(7293):1357-1361.

74. Libby P. The changing landscape of atherosclerosis. Nature. 2021;592(7855):524-533.

75. Ridker PM, et al. Antiinflammatory therapy with canakinumab for atherosclerotic disease. $N$ Engl J Med. 2017;377(12):1119-1131.

76. Tardif JC, et al. Efficacy and safety of low-dose colchicine after myocardial infarction. $N$ EnglJ Med. 2019;381(26):2497-2505.

77. Hansen SEJ, et al. Low-grade inflammation in the association between mild-to-moderate hypertriglyceridemia and risk of acute pancreatitis: a study of more than 115000 individuals from the general population. Clin Chem. 2019;65(2):321-332.

78. Yvan-Charvet L, et al. ATP-binding cassette transporters and HDL suppress hematopoietic stem cell proliferation. Science. 2010;328(5986):1689-1693.

79. Murphy AJ, et al. ApoE regulates hematopoietic stem cell proliferation, monocytosis, and monocyte accumulation in atherosclerotic lesions in mice. J Clin Invest. 2011;121(10):4138-4149.

80. Poller WC, et al. Hematopoiesis and cardiovascular disease. Circ Res. 2020;126(8):1061-1085.

81. Harslof M, et al. Low high-density lipoprotein cholesterol and high white blood cell counts: a mendelian randomization study. Arterioscler Thromb Vasc Biol. 2021;41(2):976-987.

82. Jaiswal S, et al. Clonal hematopoiesis and risk of atherosclerotic cardiovascular disease. $N$ Engl $J$ Med. 2017;377(2):111-121.

83. Fuster JJ, et al. Clonal hematopoiesis associated with Tet2 deficiency accelerates atherosclerosis development in mice. Science. 2017;355(6327):842-847.

84. Fidler TP, et al. The AIM2 inflammasome exacerbates atherosclerosis in clonal haematopoiesis. Nature. 2021;592(7853):296-301.

85. Adamstein NH, et al. The neutrophil-lymphocyte ratio and incident atherosclerotic events: analyses from five contemporary randomized trials. Eur Heart J. 2021;42(9):896-903.

86. Kashyap ML, et al. Quantitation of human apolipoprotein C-III and its subspecie by radioimmunoassay and analytical isoelectric focusing: abnormal plasma triglyceride-rich lipoprotein apolipoprotein C-III subspecie concentrations in hypertriglyceridemia. J Lipid Res. 1981;22(5):800-810.

87. Qamar A, et al. Plasma apolipoprotein C-III levels, triglycerides, and coronary artery calcification in type 2 diabetics. Arterioscler Thromb Vasc Biol. 2015;35(8):1880-1888.

88. Kanter JE, et al. Increased apolipoprotein C3 drives cardiovascular risk in type 1 diabetes. J Clin Invest. 2019;129(10):4165-4179.

89. Ito $\mathrm{Y}$, et al. Hypertriglyceridemia as a result of human apo CIII gene expression in transgenic mice. Science. 1990;249(4970):790-793.

90. Masucci-Magoulas L, et al. A mouse model with features of familial combined hyperlipidemia. Science. 1997;275(5298):391-394.
91. Gordts PL, et al. ApoC-III inhibits clearance of triglyceride-rich lipoproteins through LDL family receptors. J Clin Invest. 2016;126(8):2855-2866.

92. Ramms B, et al. ApoC-III ASO promotes tissue LPL activity in the absence of apoE-mediated TRL clearance. JLipid Res. 2019;60(8):1379-1395.

93. Pollin TI, et al. A null mutation in human APOC 3 confers a favorable plasma lipid profile and apparent cardioprotection. Science. 2008;322(5908):1702-1705.

94. TG and HDL Working Group of the Exome Sequencing Project, National Heart, Lung, and Blood Institute, et al. Loss-of-function mutations in APOC3, triglycerides, and coronary disease. N EnglJMed. 2014;371(1):22-31.

95. Jorgensen $A B$, et al. Loss-of-function mutations in APOC 3 and risk of ischemic vascular disease. N Engl JMed. 2014;371(1):32-41.

96. Saleheen D, et al. Human knockouts and phenotypic analysis in a cohort with a high rate of consanguinity. Nature. 2017;544(7649):235-239.

97. Reyes-Soffer G, et al. Effects of APOC3 heterozygous deficiency on plasma lipid and lipoprotein metabolism. Arterioscler Thromb Vasc Biol. 2019;39(1):63-72.

98. Wulff AB, et al. APOC3 loss-of-function mutations, remnant cholesterol, low-density lipoprotein cholesterol, and cardiovascular risk: mediation- and meta-analyses of 137895 individuals. Arterioscler Thromb Vasc Biol. 2018;38(3):660-668.

99. Mendivil CO, et al. Low-density lipoproteins containing apolipoprotein C-III and the risk of coronary heart disease. Circulation. 2011;124(19):2065-2072.

100. Hiukka A, et al. ApoCIII-enriched LDL in type 2 diabetes displays altered lipid composition, increased susceptibility for sphingomyelinase, and increased binding to biglycan. Diabetes. 2009;58(9):2018-2026.

101. Gaudet D, et al. Targeting APOC3 in the familial chylomicronemia syndrome. $N$ Engl J Med. 2014;371(23):2200-2206.

102. Gaudet D, et al. Antisense inhibition of apolipoprotein C-III in patients with hypertriglyceridemia. NEngl J Med. 2015;373(5):438-447.

103. Witztum JL, et al. Volanesorsen and triglyceride levels in familial chylomicronemia syndrome. N Engl J Med. 2019;381(6):531-542.

104. Fitzgerald $\mathrm{K}$, et al. A highly durable RNAi therapeutic inhibitor of PCSK9. N Engl JMed. 2017;376(1):41-51.

105.Schwabe $\mathrm{C}$, et al. RNA interference targeting apolipoprotein C-III with ARO-APOC3 in healthy volunteers mimics lipid and lipoprotein findings seen in subjects with inherited apolipoprotein C-III deficiency. Eur Heart J. 2020;41(suppl 2):ehaa946.3330.

106. Khetarpal SA, et al. A human APOC3 missense variant and monoclonal antibody accelerate apoCIII clearance and lower triglyceride-rich lipoprotein levels. Nat Med.2017;23(9):1086-1094.

107. Musunuru K, et al. Exome sequencing, ANGPTL3 mutations, and familial combined hypolipidemia. N Engl J Med. 2010;363(23):2220-2227.

108. Dewey FE, et al. Genetic and pharmacologic inactivation of ANGPTL3 and cardiovascular disease. N Engl J Med. 2017;377(3):211-221.

109. Graham MJ, et al. Cardiovascular and metabolic effects of ANGPTL3 antisense oligonucleotides. 
N Engl JMed. 2017;377(3):222-232.

110. Raal FJ, et al. Evinacumab for homozygous familial hypercholesterolemia. N Engl J Med. 2020;383(8):711-720.

111. Shimamura M, et al. Angiopoietin-like protein3 regulates plasma HDL cholesterol through suppression of endothelial lipase. Arterioscler Thromb Vasc Biol. 2007;27(2):366-372.

112. Gusarova V, et al. ANGPTL3 blockade with a human monoclonal antibody reduces plasma lipids in dyslipidemic mice and monkeys. J Lipid Res. 2015;56(7):1308-1317.

113. Adam RC, et al. Angiopoietin-like protein 3 governs LDL-cholesterol levels through endothelial lipase-dependent VLDL clearance. J Lipid Res. 2020;61(9):1271-1286.

114. Wu L, et al. LDL-cholesterol reduction by ANGPTL3 inhibition in mice is dependent on endothelial lipase. Circ Res. 2020;127(8):1112-1114.

115. Ishida $T$, et al. Endothelial lipase modulates susceptibility to atherosclerosis in apolipoprotein-E-deficient mice. J Biol Chem. 2004;279(43):45085-45092.

116. Ko KW, et al. Endothelial lipase modulates HDL but has no effect on atherosclerosis development in apoE-/- and LDLR-/- mice. J Lipid Res. 2005;46(12):2586-2594.

117. Pisciotta $\mathrm{L}$, et al. Characterization of three kindreds with familial combined hypolipidemia caused by loss-of-function mutations of ANGPTL3. Circ Cardiovasc Genet. 2012;5(1):42-50.

118. Tall AR. Increasing lipolysis and reducing atherosclerosis. N Engl J Med. 2017;377(3):280-283.

119. Gaudet D, et al. Vupanorsen, an $\mathrm{N}$-acetyl galactosamine-conjugated antisense drug to ANGPTL3 mRNA, lowers triglycerides and atherogenic lipoproteins in patients with diabetes, hepatic steatosis, and hypertriglyceridaemia. Eur Heart J. 2020;41(40):3936-3945.

120. Dewey FE, et al. Inactivating variants in ANGPTL4 and risk of coronary artery disease. N Engl J Med. 2016;374(12):1123-1133.

121. Lichtenstein L, et al. Angptl 4 protects against severe proinflammatory effects of saturated fat by inhibiting fatty acid uptake into mesenteric lymph node macrophages. Cell Metab. 2010;12(6):580-592.

122. Desai U, et al. Lipid-lowering effects of anti-angiopoietin-like 4 antibody recapitulate the lipid phenotype found in angiopoietin-like 4 knockout mice. Proc NatlAcad Sci U S A. 2007;104(28):11766-11771.

123. Singh AK, et al. Hepatocyte-specific suppression of ANGPTL4 improves obesity-associated diabetes and mitigates atherosclerosis in mice. J Clin Invest. 2021;131(17):e140989.

124. Shearer GC, et al. Fish oil - how does it reduce plasma triglycerides? Biochim Biophys Acta. 2012;1821(5):843-851.

125. Yokoyama M, et al. Effects of eicosapentaenoic acid on major coronary events in hypercholesterolaemic patients (JELIS): a randomised open-label, blinded endpoint analysis. Lancet. 2007;369(9567):1090-1098.

126. Saito Y, et al. Effects of EPA on coronary artery disease in hypercholesterolemic patients with multiple risk factors: sub-analysis of primary prevention cases from the Japan EPA Lipid Intervention Study (JELIS). Atherosclerosis. 2008;200(1):135-140.
127. ASCEND Study Collaborative Group, et al. Effects of n-3 fatty acid supplements in diabetes mellitus. N EnglJ Med. 2018;379(16):1540-1550.

128. Nicholls SJ, et al. Effect of high-dose omega-3 fatty acids vs corn oil on major adverse cardiovascular events in patients at high cardiovascular risk: the STRENGTH Randomized Clinical Trial. JAMA. 2020;324(22):2268-2280.

129. Kastelein JJP, Stroes ESG. FISHing for the miracle of eicosapentaenoic acid. N Engl J Med. 2019;380(1):89-90.

130. Doi T, et al. A possible explanation for the contrasting results of REDUCE-IT vs. STRENGTH: cohort study mimicking trial designs [published online August 29, 2021]. Eur Heart J. https://doi. org/10.1093/eurheartj/ehab555.

131. Hu Y, et al. Marine omega-3 supplementation and cardiovascular disease: an updated meta-analysis of 13 randomized controlled trials involving 127477 participants. J Am Heart Assoc. 2019;8(19):e013543.

132. Fisher EA, et al. The triple threat to nascent apolipoprotein B. Evidence for multiple, distinct degradative pathways. J Biol Chem. 2001;276(30):27855-27863.

133. Back M, et al. Inflammation and its resolution in atherosclerosis: mediators and therapeutic opportunities. Nat Rev Cardiol. 2019;16(7):389-406.

134. Blom DJ, et al. Lomitapide and mipomerseninhibiting microsomal triglyceride transfer protein (MTP) and apoB100 synthesis. Curr Atheroscler Rep. 2019;21(12):48.

135. Fruchart JC, et al. Selective peroxisome proliferator-activated receptor alpha modulators (SPPAR$\mathrm{M} \alpha)$ in the metabolic syndrome: is pemafibrate light at the end of the tunnel? Curr Atheroscler Rep. 2021;23(1):3.

136. Nordestgaard BG, Varbo A. Triglycerides and cardiovascular disease. Lancet. 2014;384(9943):626-635.

137. Westerterp M, et al. Deficiency of ATP-binding cassette transporters $\mathrm{A} 1$ and $\mathrm{G} 1$ in macrophages increases inflammation and accelerates atherosclerosis in mice. Circ Res. 2013;112(11):1456-1465.

138. Westerterp M, et al. Deficiency of ATP-binding cassette transporters $\mathrm{A} 1$ and $\mathrm{G} 1$ in endothelial cells accelerates atherosclerosis in mice. Arterioscler Thromb Vasc Biol. 2016;36(7):1328-1337.

139. Rohatgi A, et al. HDL cholesterol efflux capacity and incident cardiovascular events. N EnglJ Med. 2014;371(25):2383-2393.

140.El-Ghazali A, et al. Molecular patterns of extreme and persistent cholesterol efflux capacity. Arterioscler Thromb Vasc Biol. 2021;41(10):2588-2597.

141. Shea S, et al. Cholesterol mass efflux capacity, incident cardiovascular disease, and progression of carotid plaque. Arterioscler Thromb Vasc Biol. 2019;39(1):89-96.

142.Garg PK, et al. Cholesterol mass efflux capacity and risk of peripheral artery disease: the Multi-Ethnic Study of Atherosclerosis. Atherosclerosis. 2020;297:81-86.

143. Jia C, et al. High-density lipoprotein anti-inflammatory capacity and incident cardiovascular events. Circulation. 2021;143(20):1935-1945.

144. Hong C, Tontonoz P. Liver X receptors in lipid metabolism: opportunities for drug discovery. Nat Rev Drug Discov. 2014;13(6):433-444.

145. Kirchgessner TG, et al. Beneficial and adverse effects of an LXR agonist on human lipid and lipoprotein metabolism and circulating neutrophils. Cell Metab. 2016;24(2):223-233.

146.Zelcer N, et al. LXR regulates cholesterol uptake through Idol-dependent ubiquitination of the LDL receptor. Science. 2009;325(5936):100-104.

147. Yu M, et al. Targeted nanotherapeutics encapsulating liver X receptor agonist GW3965 enhance antiatherogenic effects without adverse effects on hepatic lipid metabolism in $\mathrm{Ldll}^{-/}$mice. Adv Healthc Mater. 2017;6(20):1700313.

148.HPS3/TIMI55-REVEAL Collaborative Group, et al. Effects of anacetrapib in patients with atherosclerotic vascular disease. $N$ Engl JMed. 2017;377(13):1217-1227.

149. Tall AR, Rader DJ. Trials and tribulations of CETP inhibitors. Circ Res. 2018;122(1):106-112.

150.Badimon JJ, et al. Regression of atherosclerotic lesions by high density lipoprotein plasma fraction in the cholesterol-fed rabbit. J Clin Invest. 1990;85(4):1234-1241.

151. Rubin EM, et al. Inhibition of early atherogenesis in transgenic mice by human apolipoprotein AI. Nature. 1991;353(6341):265-267.

152. Plump AS, et al. Human apolipoprotein A-I gene expression increases high density lipoprotein and suppresses atherosclerosis in the apolipoprotein E-deficient mouse. Proc Natl Acad Sci U S A. 1994;91(20):9607-9611.

153. Murphy AJ, et al. Pegylation of high-density lipoprotein decreases plasma clearance and enhances antiatherogenic activity. Circ Res. 2013;113(1):e1-e9.

154.Fotakis P, et al. Anti-inflammatory effects of HDL (high-density lipoprotein) in macrophages predominate over proinflammatory effects in atherosclerotic plaques. Arterioscler Thromb Vasc Biol. 2019;39(12):e253-e272.

155. Tardif JC, et al. Effects of reconstituted high-density lipoprotein infusions on coronary atherosclerosis: a randomized controlled trial. JAMA. 2007;297(15):1675-1682.

156. Gibson CM, et al. Rationale and design of ApoA-I Event Reducing in Ischemic Syndromes II (AEGIS-II): a phase 3, multicenter, double-blind, randomized, placebo-controlled, parallel-group study to investigate the efficacy and safety of CSL112 in subjects after acute myocardial infarction. Am Heart J. 2021;231(aegis-ii):121-127.

157. Gibson CM, et al. Safety and tolerability of CSL112, a reconstituted, infusible, plasma-derived apolipoprotein A-I, after acute myocardial infarction: the AEGIS-I trial (ApoA-I Event Reducing in Ischemic Syndromes I). Circulation. 2016;134(24):1918-1930.

158. Gibson CM, et al. Rationale and design of Apo-I Event Reduction in Ischemic Syndromes I (AEGIS-I): a phase $2 \mathrm{~b}$, randomized, placebo-controlled, dose-ranging trial to investigate the safety and tolerability of CSL112, a reconstituted, infusible, human apoA-I, after acute myocardial infarction. Am Heart J. 2016;180 (aegis-i):22-28.

159. Goldstein JL, et al. Hyperlipidemia in coronary heart disease. I. Lipid levels in 500 survivors of myocardial infarction. J Clin Invest. 1973;52(7):1533-1543.

160.Zilversmit DB. Atherogenic nature of triglycerides, postprandial lipidemia, and triglyceride-rich remnant lipoproteins. Clin Chem. 1995;41(1):153-158. 